\title{
AN ANALYSIS OF IMAGE COMPRESSION TECHNIQUES IN WIRELESS MULTIMEDIA SENSOR NETWORKS
}

\author{
Arafat Senturk, Resul Kara
}

Subject review

Wireless Multimedia Sensor Networks (WMSNs) provide realization of applications which are usable everywhere and address many fields like mobile health care, environmental surveillance and traffic monitoring. Large amount of data causes to traffic in memory resources, difficulties in operation, and excessive power consumption -which is the most important one - for every node while WMSNs transfer multimedia data during those applications. Those kinds of problems are vital for WMSNs which already have limited resources. Image compression can be one of the effective solutions to overcome those problems. Thus, network lifetime of WMSNs can be increased significantly and the bandwidth can be used in a more effective way. The main purpose of this study is to investigate image compression algorithms used for WMSNs in the literature and to show which algorithm is advantageous in which case by making comparisons among them.

Keywords: energy efficiency; image compression; wireless multimedia sensor network

\section{Analiza načina sažimanja slike u bežičnoj višemedijskoj senzorskoj mreži}

Pregledni članak Bežične višemedijske senzorske mreže - Wireless Multimedia Sensor Networks (WMSNs) omogućuju realizaciju aplikacija koje se svugdje mogu primijeniti, a odnose se na mnoga područja kao što je briga o zdravlju, praćenje stanja okoliša i upravljanje prometom. Izmjena velikih količina podataka stvara poteškoće u funkcioniranju memorije te prekomjernu potrošnju energije u svakom čvoru - a to je najvažnije - dok WMSNs prenose multimedijske podatke tijekom tih aplikacija. Ta je vrsta problema od bitne važnosti za WMSNs koji već imaju ograničene resurse. Sažimanje slike može biti jedno od učinkovitih rješenja za svladavanje tih problema. Trajnost WMSNs na mreži može se tako značajno povećati, a širina područja se može učinkovitije iskoristiti. Glavna svrha ovoga rada je istražiti algoritme sažimanja slike koji se u literaturi primjenjuju za WMSNs te uspoređujući ih pokazati koji algoritam ima prednost u pojedinom slučaju.

Ključne riječi: bežična multimedijska senzorska mreža; sažimanje slike; učinkovitost energije

\section{Introduction}

Wireless Sensor Networks (WSNs) are the networks which realize data processing and computation features of sensor nodes on wireless channel with several communication devices $[1,2]$. As for Wireless Multimedia Sensor Networks (WMSN), they are the networks which transfer real-time multimedia data (sound, image, and video) to each other or to a sink via sensors with cheap hardware. Audio and visual data may exist on a single device. Besides transmission, WMSNs are able to store real-time data after taking it from many sensors.

Besides their usage in some applications like traffic control systems, advanced healthcare services and industrial process control, WMSNs are mostly used for monitoring purposes. Surveillance systems are developed against crime and terrorism attacks by using video and audio sensors. Since wireless nodes and video cameras in WMSN are capable of high computation, they facilitate several applications [3, 4]. Furthermore, classification of the images taken from WMSN increases the usability of the application significantly [5].

Recently, CMOS cameras and microphones which have cheap hardware are started to be used to capture multimedia content (video and sound flow) around. Audio and video contents exist together in a single device thanks to the developments in hardware [6]. The most promising developments in real-time multimedia monitoring are obtained with WMSN. But, some resource requirements of the applications like network lifetime and energy become important issues in WMSN since the data amount to be transferred is rather more than WSN [1, 7]. Power consumption is a fundamental issue for WMSN. It uses most of the energy for data transmission. Therefore, a proper design is required to maximize WMSN lifetime. Other problems that affect the development of WMSN are limited band width and memory $[1,8]$.

WMSN needs high memory to store multimedia data and high band width to transfer high resolution images. It is required for an image which is captured by a sensor node to be compressed before processing and transmission because of limited band width. An effective method can be provided via image compression by removing redundant data. In addition, compressing the images reduces the cost of storage [9].

WMSNs can be evaluated in three phases: sensing, data processing and transmission. Data processing and transmission phases are the phases which incur energy issues. Proper compression algorithms will increase the lifetime of the sensors by decreasing data size and power consumption. Power consumption is the main factor that affects the usability of WMSNs. That is why simple compression operations must be used to eliminate energy consumption issues [1].

In the following sections, image compression algorithms for WMSN will be mentioned. Then, these algorithms will be compared and a decision will be made about which algorithm is more effective in which case.

\section{Image compression techniques in WMSNs}

Using some image compression algorithms like JPEG and JPEG2000 is not logical for WMSNs since they have limited power and band width. The most problematic cases with these algorithms are algorithm size, processor 
speed and memory access [1, 10]. Image compression techniques for WMSNs are schematically shown in Figure 1.

Image compression can be done in two ways: with data loss and without data loss. Lossless compression is generally used for the images with low correlation between pixels, while lossy techniques are used for complex and high-correlation images like camera images. Lossy techniques have important advantages such as shorter encoding/decoding time, high compression ratio and usability in power-limited applications. Sizes of the images which are compressed by lossless techniques are quite big with respect to lossy techniques and they consume more power and band width in sensor nodes.
Therefore, lossless algorithms are not preferred to transfer images on WMSNs [9].

\subsection{Lossless image compression techniques}

Lossless compression techniques are divided into two categories: Decorrelation and entropy coding [11].

\subsubsection{Decorrelation}

Decorrelation is one of the lossless techniques which remove redundancies between pixels. It can be categorized as prediction-based techniques [12], transform-based techniques and multi resolution based techniques [13].

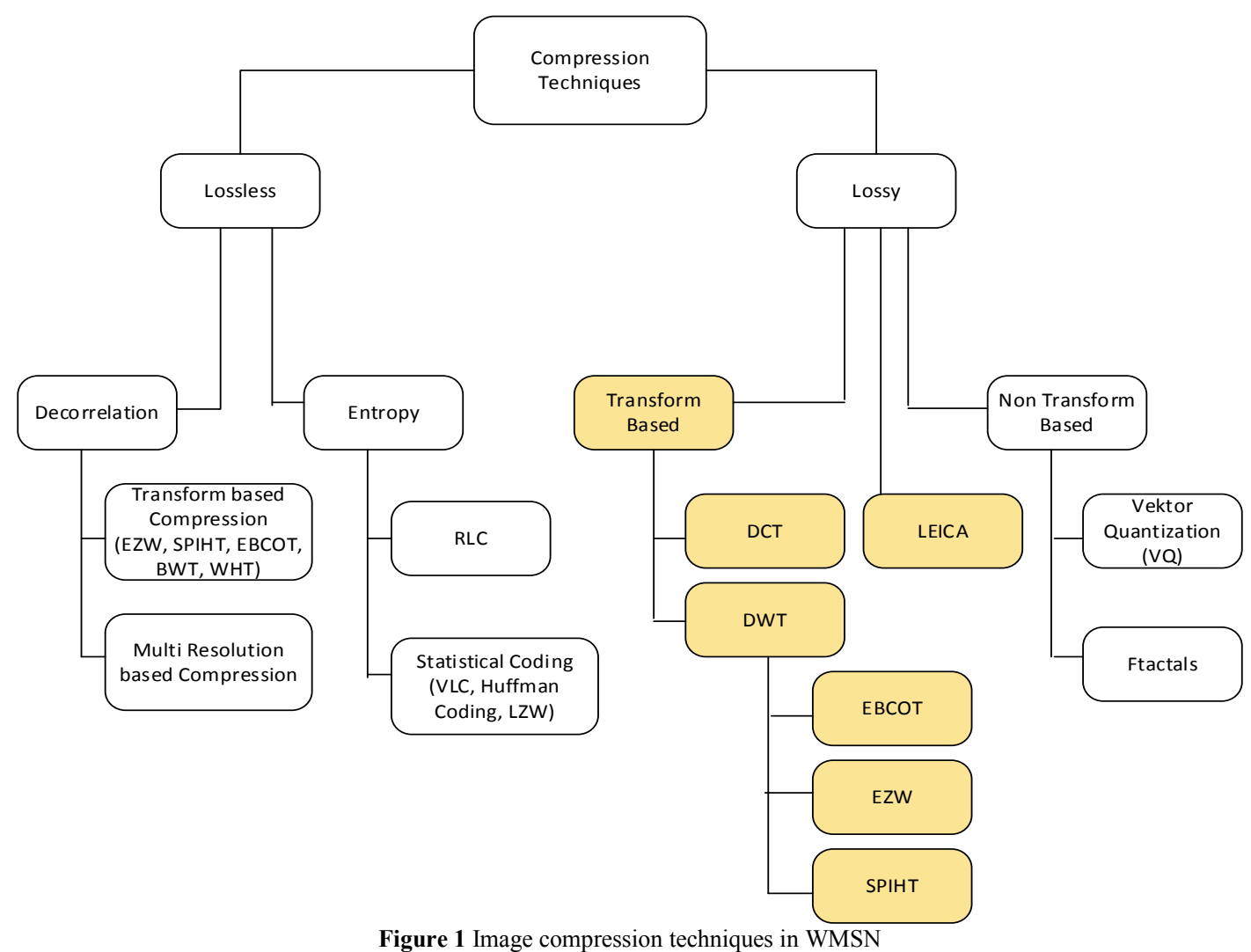

\subsubsection{Entropy coding}

Entropy coding also removes redundancies and it is based on RLC (Run Length Coding) and statistical coding. It can be used together with some lossy compression algorithms like JPEG. Lossless compression techniques never lose any data that will cause image transmission to be very complex and will reduce compression ratio.

\subsection{Lossy image compression techniques}

Lossy compression techniques have higher compression ratio than the lossless techniques. In lossy compression, the compressed image is not usually the same with the original image. Therefore, a distortion metric is used. Distortion metric is a mathematical magnitude which defines an approximation to the original image. The most frequently used distortion metrics are MSE and PSNR [9].

\subsubsection{Discrete cosine transforms (DCT)}

Discrete Cosine Transform (DCT) is the most prevalent transform coding technique and a well-known DCT-based compression scheme is JPEG [9]. DCT based image compression divides images into $8 \times 8$ blocks and encodes each block individually [17]. The equation of two dimensional DCT is the following:

$F(u, v)=$

$=\frac{1}{4} C(u) C(v) \sum_{x=0}^{k-1} \sum_{y=0}^{k-1} f(x, y) \cos \left(\frac{(2 x+1) u \pi}{2 k}\right) \cos \left(\frac{(2 y+1) v \pi}{2 k}\right),{ }^{(1)}$

$C(z)=\left\{\begin{array}{l}\frac{1}{\sqrt{2}} \text { if } z=0 \\ 1 \text { otherwise }\end{array}\right.$. 
Matrices are composed so. Matrix $\boldsymbol{P}$ is obtained by subtracting 128 from each element of the matrix. Then, matrix A and its transpose are multiplied by this matrix $\boldsymbol{P}$, and DCT matrix is found.

$$
\boldsymbol{F}(k \times k)=\boldsymbol{A}(k \times k) \boldsymbol{P}(k \times k) \boldsymbol{A}^{\mathrm{T}}(k \times k) .
$$

Next, quantization operation occurs. How much the image will be compressed is determined thanks to this operation optionally. Quantization formula is the following:

$$
C_{i, j}=\operatorname{round}\left(\frac{D_{i, j}}{Q_{i, j}}\right) \text {. }
$$

Lossy JPEG compression scheme is given in Fig. 2.

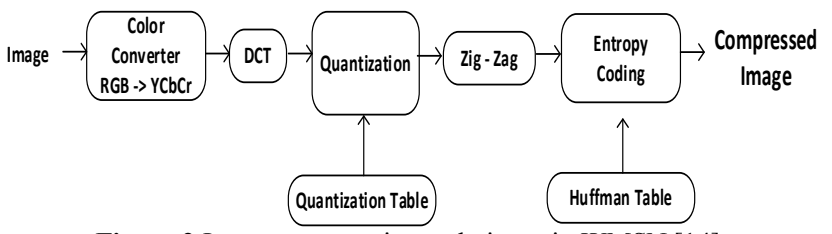

Figure 2 Image compression techniques in WMSN [14]

DCT based image compression provides sufficient compression efficiency and encoding is done with small individual image blocks. Thus, low-memory applications can be realized using it [17].

\subsubsection{Discrete wavelet transforms (DWT)}

Image compression with wavelet functions are one of the transform based compression methods. First, wavelet transform is applied to the image and some of the coefficients are removed from the image. The decision about which coefficients will be thrown or which of them will be saved, can be given according to application such as statistical calculations, signal energy position of the image and experienced threshold value. Encoding is applied on the rest of the coefficients. Compressed image is reconstructed back from the decoded coefficients and the original image is obtained by applying inverse transform [18].

The fundamental idea in wavelet analysis is to reacquire information from the details in different levels. Wavelet transform can be considered as a tree scheme which is composed of high-pass and low-pass filters. While low-pass filter $\{\mathrm{Ln}\}$ is reducing the information in signal or in a data array, high-pass filter $\{\mathrm{Hn}\}$ saves this lost information. The image is separated into average sub bands with wavelet transform [9].

Most of the energy density of an image is generally intensified in low-frequency regions. Spectral components of an ordinary image decreases as the frequency increases. This feature of an image is seen in DWT, too. Assume that n-level DWT decomposition is done. In this case, low levels correspond to high frequency sub bands. For example, while the first level shows the highest frequency sub band, at the same time it shows a level in which the resolution is the highest. Therefore, the nth level shows the lowest frequency sub band and corresponds to the lowest resolution level. So, energy content of lower band decreases as we go from higher levels to lower levels. Similarly, when the pixel magnitude value of a sub band in a certain level is observed as low, the pixel value of a sub band in a lower level is expected to be lower [18].

Wavelet based transforms present a signal in time and frequency domains with a well resolution by using a series of basic functions called wavelet. Wavelets in image compression are separable functions. To apply these, first a low-pass filter is applied on the rows to generate $\mathrm{L}$ and $\mathrm{H}$ sub bands, and then LL, LH, HL, and $\mathrm{HH}$ sub bands are generated by applying high-pass filter to the columns. In the second step, each of the four sub bands is separated into four sub bands as LL2, LH2, HL2, and $\mathrm{HH} 2$ and so on. LL sub band is shown in Figure 3 [19].

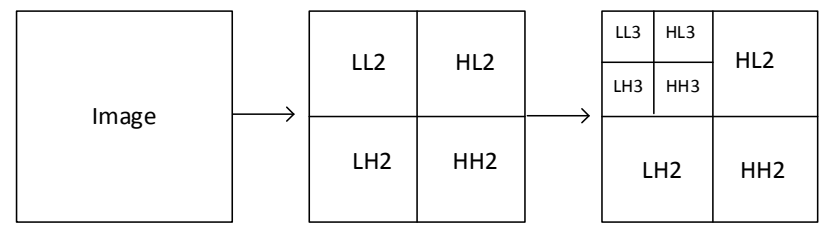

1. Level 2. Level

Figure 3 2-level 2D DWT decomposition [19]

\subsubsection{Embedded zero-tree wavelet (EZW) based Compression technique}

Embedded Zero-tree Wavelet is a simple and effective image compression algorithm and its property is that bits in a bit array are generated based on their importance. As a result, a fully embedded code arises and this embedded code is an array of binary decisions which distinguish the image from an empty image. An encoder may stop encoding at any point and so the target rate or target distortion metric is satisfied. Furthermore, given a bit array, decoder may stop decoding at any point and again the same image with a bit rate which corresponds to the clipped bit array is generated. In addition to generating fully embedded bit array, EZW produces such compression results that may compete with many wellknown compression algorithms. This performance is achieved by a technique which does not require training, preloaded tables, code books or previously known resource images [20].

EZW is based on four concepts [20]:

- Discrete wavelet transform

- $\quad$ Predicting absence of important information by using similar images

- Successive prediction scaling with entropy coding

- "Universal" lossless data compression which is achieved by adaptive arithmetic encoding

\subsubsection{Set partitioning in hierarchical trees (SPIHT)}

Set partitioning in hierarchical trees (SPIHT) is an advanced version of EZW and a powerful wavelet based compression algorithm [21]. To compress an image with SPIHT, wavelet transform is applied to the image before partitioning. The sub bands which are obtained by wavelet transform have small-value coefficients that 
compose a few percentages of the image energy. Quantifying these small-value coefficients to zero does not cause too serious losses in the image. The image which is constituted with this operation has mostly zerovalue pixels. Coding steps of SPIHT are given in Fig. 4.

This property of wavelet transform is the basis of SPIHT. DWT of the images show an unstable characteristic. In other words, pixel values of detailed images are mostly composed of zeros or the values close to zero. The coefficients of DWT in this algorithm are organized as spatial orientation tree. The reason of constituting these trees is to collect high-correlation pixels in a set. Coefficient in a level and similarity rate in another level is increased with this set configuration. Thanks to this feature of wavelet transform, high compression ratio in images is obtained with SPIHT.

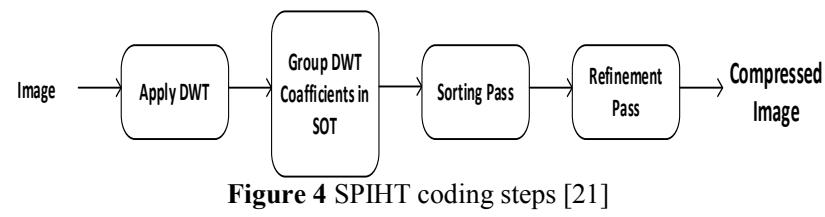

\subsubsection{Low energy image compression algorithm (LEICA)}

Image is divided into two parts as Region of Interest (ROI) and background. ROI is the region which is expected to be seen. To save image quality, the image is compressed with low compression ratio. Background is assumed to be less important and so, this part can be compressed more. Thus, the energy of the image is saved more [23].

Camera nodes keep background and save it into the memory when the monitored object is not seen. Scalar sensor node is used for monitoring objects. If the object is not seen, then the camera which is stays in passive mode. Otherwise, the camera triggered by the scalar sensor node captures the image and the captured image is defined as $f$. The difference of each block between the captured image $(f)$ and the background $(b)$ is calculated by the formula given below. Camera node goes on taking background image. $\rho$ value in each block is calculated as given below [23].

$$
D_{m, n}=\frac{1}{64} \sum_{i=1}^{8} \sum_{j=1}^{8}|f(8 m+i, 8 n+j)-b(8 m+i, 8 n+j)|
$$

(5)

$$
\begin{aligned}
& m=0,1, \ldots, M / 8-1 ; n=0,1, \ldots, M / 8-1 \\
& \rho_{m, n}=\left\{\begin{array}{ll}
\rho_{\max }, & \text { if } D_{m, n}>D_{0} \\
\rho_{\min }, & \text { otherwise }
\end{array} .\right.
\end{aligned}
$$

The value of $D_{0}$, which is the threshold value between ROI and the blocks, is determined by camera node and the monitored scene. Image quality of ROI determines $P_{\text {max }}$. The value of $P_{\min }$ is determined by the remaining energy of the nodes and image quality of the pixels. The value of $P_{\min }$ is less than the value of $P_{\max }$. When an image is compressed, the value of $P$ min determines the number of DCT coefficients. Other operations of LEICA are the same as JPEG [23].

\subsubsection{Embedded block coding with optimized truncation (EBCOT)}

Embedded block coding with optimized truncation (EBCOT) is a block based coding algorithm. The main idea of EBCOT is to transform LL, LH, HL, and HH sub bands into smaller code blocks. Each block is coded independently [5]. EBCOT is divided into two parts: data coding and data arrangement. Block diagram of JPEG2000 is given in Figure 5. Embedded coding is realized as block based. A popular image compression standard, JPEG2000, adopts EBCOT compression technique [24].

EBCOT is realized as a new type of scalable image compression techniques which is done previously. It uses wavelet transform to generate sub band samples which are able to be coded after quantifying. Scalable compression corresponds to generating a bit array that consists of embedded sub sets. Each of these subsets show the effectively compressed version of the original image whose resolution is decreased or distortion ratio is increased. Resolution scalability and SNR scalability terms appear at this point. A bit array is called as resolution scalable if it consists of discrete sub sets each of which shows subsequent resolution level. If a bit array consists of discrete sub bands which show all sub bands in a certain quality (SNR) level, SNR of that bit array is scalable. The most important advantage of scalable compression is that there is no need to know about the target bit rate or resolution during compression. Another advantage is that there is no need to compress an image more than once to achieve desired bit rate as opposed to JPEG2000 [25].

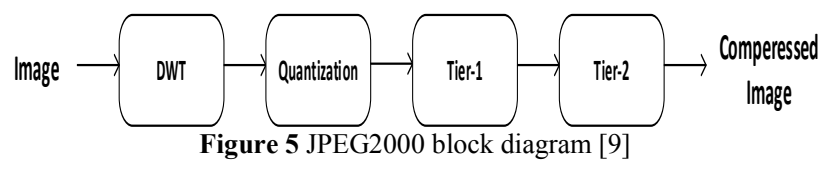

\section{Comparison of image compression techniques}

To determine which compression technique is the best one for WMSN, we may analyze the data given in Tab. 1. Although DCT requires low memory in applications when it is compared by DWT, it is not a good choice for WMSNs. It consumes approximately $60 \%$ of total energy because of the complexity of encoder and this is very much [9]. Ma et al. [33] showed that the floating point application of JPEG has the highest work load. Compilation time $(103,12 \mathrm{~ms})$ consumed most of the energy of encoder. Also, there are many efforts to reduce work load of DCT. Fakhari et al. [18] developed highefficiency architecture for DCT by using virtex 5 parallelism and consequent series on Xilinx FPGA. Additionally, Zheng and Liu [27] developed a fast DCT algorithm based on JPEG [28]. They used binary DCT system on JPEG encoder. They construct all operations on a binary system and accelerated operation speed.

There are several techniques based on discrete wavelet transform to provide energy efficiency in WMSNs. Ghorbel et al. [26] made a performance analysis for DCT and DWT. As a result, they proved that DWT is 
better than DCT in terms of image quality and energy consumption. Image quality for Lena $(64 \times 64)$ is found as 33,55 db (PSNR) with DWT and 30,52 db with DCT. Furthermore, they used AA-NiMH $2850 \mathrm{~mA}$ Alkali battery in the system. This battery is used for 281,34 hours for DWT and 245,20 hours for DCT.

Nasri et al. [29] proposed an adaptive image compression structure (skipped high-pass sub bandSHPS) which minimizes computation and communication energies. They ignored high pass coefficients which have very small values. Skipping the least significant sub band reduces work load and saves the energy. Another technique called as Elimination High-Pass Filter (EHPF) [30] is developed after that. Only low-pass filter is applied in vertical direction, not high-pass filter. Therefore, only LL and LH sub bands, which include more important information about the image, are generated. They actually provided some saving from the energy by causing little distortion in the image. This technique constitutes an adaptive compression technique, AWICAO (Adaptive wavelet image compression and organization algorithm). The selection of the most effective technique is done according to the trade-offs like energy consumption and image quality.

EBCOT is an algorithm which has the highest performance in terms of compression quality. Tier-1 operation in this algorithm is responsible for arithmetic coding and context arrangement. The encoder consumes the power very much in EBCOT during the operation. Therefore, energy consumption is very high, execution time is long because of computation complexity, and memory need appears. Thus, EBCOT is not usable for WMSNs [25]. Tier-1 operation consumes more than $70 \%$ of the energy after DWT step [19].

Jackson et al. [31] compares image compression techniques. When they compare fractal and JPEG, they see that fractal has bigger compression rate, but it is concluded that it is not that much useful since it decreases the lifetime of WMSN significantly by consuming large amount of energy during encoding [16, 19].

Generally, JPEG or JPEG2000 compression standards are used to save band width and storage while a visual data is being transferred. However, computation operations of those standards consume large amount of energy. WMSNs are energy-limited networks, and therefore less energy-consuming algorithms must be used [32].

SPIHT became the best solution for WMSNs because of high compression ratio, less computation complexity (than EZW [20] and DCT), and lower power consumption [9]. Ma et al. developed a version of SPIHT [33]. They improved PSNR value (between $0,2 \div 0,4$ ) with different techniques, but not using arithmetic coding. However, increasing complexity requires more memory space for SPIHT. At the end of the studies, it is deduced that SPIHT consumes very small amount of energy and has high compression ratio. In addition, Sun et al. [34] developed a real-time application based on SPIHT, which uses DSP chip. DSP chip lowered encoding time to less than $40 \mathrm{~ms}$ and reduced memory cost $13 \div 14$ times.

Kose et al. [35] compared SPIHT and JPEG compression techniques on 3D images. They showed that SPIHT outperforms according to the results of the simulations. Also, Chong et al. [36] used SPIHT to compress multiple images in WMSNs. In their analysis, the sensor nodes which will join image processing have higher computation power than the other nodes. Image sensors first capture the image, and send it to the microprocessor to make operations on it later and to remove redundancies. Next, the stitched image is compressed by SPIHT to reduce transferred bit number. Simulations show that the transferred data is $10 \div 15 \%$ reduced.

Fu-Xiao et al. [37] uses SPIHT in three phases to minimize visual distortions in WMSNs. In the first phase, wavelet transform is applied to the images. Wavelet coefficients of SPIHT are calculated in phase two, and lastly, context based arithmetic encoding is realized for SPIHT. At the end, it is mentioned that SPIHT is useful since it minimizes image distortions in WMSNs.

EBCOT provides high-efficiency compression when it is compared by SPIHT, but there are multi-layered coding operations in EBCOT. Besides, it needs multiple encoding tables for arithmetic encoding, and this makes the system more complex and expensive (because of extra memory requirement). So, SPIHT comes into prominence [24, 38].

The image is divided into two parts as background and foreground in LEICA. The background is assumed to be unimportant and high compression ratio is realized there. Therefore, LEICA is not appropriate for the situations in which both background and foreground are of the same importance. Also, in some applications, sensor nodes are required to be always awake, but in LEICA sensors are passive when no object is seen. It is seen that LEICA is quite efficient in terms of computation complexity and power consumption. LEICA may be useful towards the needs of the application [23].

Table 1 Evaluation of the mentioned image compression algorithms

\begin{tabular}{|c|c|c|c|c|c|}
\hline & DCT [9, 16, 17] & EZW [20] & EBCOT [21, 24] & SPIHT [22] & LEICA [23] \\
\hline Memory Requirement & Low & Medium & High & Medium & Medium \\
\hline Operation complexity & Low & Low & High & Medium & Medium \\
\hline Power consumption & Low & Medium & High & How \\
\hline Compression ratio & Low & Medium & High & High & High \\
\hline Compressed image quality & Low & Low & High & Medium & Medium \\
\hline System complexity & Low & Medium & Medium & High & High \\
\hline Coding speed & High & Low & Medium & High & High \\
\hline
\end{tabular}

\section{Evaluation of image compression techniques}

After the evaluations of prevalent image compression techniques in WMSNs, the following results can be obtained:
- $60 \%$ of total energy is consumed during encoding in DCT compression algorithm. PSNR value is lower than DWT-based techniques. It is found to be 30,52 $\mathrm{db}$ in Lena application, although it is 33,55 in DWTbased techniques [25]. 
- EZW is one of the very first algorithms used in WMSNs. The system, which is based on the prediction of the absence of important information by making use of similar images, contains complexness although it provides high compression ratio [20].

- $\quad 70 \%$ of total energy is consumed during encoding when EBCOT compression algorithm is used [11].

- Computation speed of SPIHT is very high and it may improve image quality by $0,2 \div 0,4$ rates [18]

- Number of dead nodes in LEICA is very small when compared by other techniques. Also, network lifetime is $87 \%$ longer than JPEG [23].

The properties of the above mentioned algorithms are comparatively given in Tab. 1 .

\section{Conclusion}

Image compression techniques are very important in WMSNs which have many hardware and energy limitations. Therefore, the preferred method must have the features like fast and efficient image processing, low memory requirement, high compression quality, noncomplex system, and low computation load.

In this study, all image compression algorithms that can be used for WMSNs are investigated and they are briefly compared in Tab. 1. According to this analysis, a compression technique can be chosen considering the specifications of the application. Some selection priorities for three efficient image compression techniques can be the followings:

- DCT for the situations in which the image quality is expected to be adjusted.,

- LEICA for the situations in which nodes become optionally awaked and image quality is ignored.

- SPIHT for high compression ratio and energy efficiency.

It is concluded that using specific algorithms in specific situations provides efficiency in WMSNs.

In the next study, it is planned to develop such an application that chooses a proper algorithm dynamically according to system requirements. This feature will be provided automatically. It is predicted that energy efficiency of the system will reach its maximum with this approach.

\section{References}

[1] Akyildiz, I. F.; Melodia, T.; Chowdhury, K. R. A survey on wireless multimedia sensor networks. // Computer Networks. 51, 4(2007), pp. 921-960. DOl: 10.1016/j.comnet.2006.10.002

[2] Magli, E.; Mancin, M.; Merello, L. Low-complexity video compression for wireless sensor networks. // Proceedings in International Conference on Multimedia and Expo. (2003), pp. 585-588. DOI: 10.1109/icme.2003.1221379

[3] Akyildiz I. F.; Melodia T.; Kaushik R. C. Wireless Multimedia Sensor Networks: Applications Networks and Testbeds. // Proceedings of the IEEE. 96, 10(2008), pp. 1588-1605. DOI: 10.1109/JPROC.2008.928756

[4] Harjito, B.; Han, S. Wireless Multimedia Sensor Networks Applications and Security Challenges. // International Conference on Broadband, Wireless Computing,
Communication and Applications / Fukuoka, 2010, pp. 842-846. DOI: 10.1109/bwcca.2010.182

[5] Wang, X.; Wang, S.; Bi, D. Compacted Probabilistic Visual Target Classification with Committee Decision in Wireless Multimedia Sensor Network. // IEEE Sensors Journal. 9, 4(2009), pp. 346-353. DOI: 10.1109/JSEN.2009.2013917

[6] Rahimi, M.; Baer, R.; Iroezi, O. I.; Garcia, J.C.; Warrior, J.; Estrin D.; Srivastava, M. Cyclops: in situ image sensing and interpretation in wireless sensor Networks. // Proceedings of the ACM International Conference on Embedded Networked Sensor Systems / USA, (2005), pp. 192-204. DOI: 10.1145/1098918.1098939

[7] Cesana, M.; Redondi, A.; Tiglao, N.; Grilo, A.; BarceloOrdinas, J. M.; Alaei M.; Todorova, P. Real-time Multimedia Monitoring in Large-Scale Wireless Multimedia Sensor Networks: Research Challenges. $/ / 8^{\text {th }}$ Euro-NF Conference on Next Generation Internet (NGI) / Karlskrona, (2012), pp. 79-86. DOl: 10.1109/ngi.2012.6252168

[8] Mainwaring, A.; Polastre, J.; Szewczyk, R.; Culler, D.; Anderson, J. Wireless sensor networks for habitat monitoring. // Proceedings of the $1^{\text {st }}$ ACM international workshop on Wireless sensor networks and applications USA, (2002), pp. 88-97. DOI: 10.1145/570738.570751

[9] Chew, L. W.; Ang, L. M.; Seng, K. P. Survey of image compression algorithms in wireless sensor networks. // International Symposium on Information Technology / Malaysia, (2008), pp. 1-9.

[10] Ghorbel, O.; Jabri, I.; Ayedi, W.; Abid M. Experimental study of compressed images transmission through WSN. // Microelectronics (ICM) International Conference / Hammamet, (2011), pp. 1-6.

[11] Yang, M.; Bourbakis, N. An overview of lossless digital image compression techniques. // IEEE Circuit system $48^{\text {th }}$ Midwest Symposium / 2005, pp. 1099-1102.

[12] Shukla, J.; Alwani, M.; Tiawari A. K. A survey on lossless image compression methods. // Computer engineering and technology (ICCET) IEEE $2^{\text {nd }}$ international conference / Chengdu, 2010, pp. 136-141.

[13] Carreto-Castro, M. F.; Ramirez, J. M.; Ballesteros, J. L.; Baez-Lopez, D. Comparison of lossless compression techniques // Circuits and systems proceedings of the $36^{\text {th }}$ midwest symposium / Detroit, MI, 1993, pp. 1268-1270.

[14] Li, Z. N.; Drew, M. S.; Liu, J. Image compression standards in Fundamentals of multimedia. Prentice-Hall, Springer, 2004

[15] Samra, H. S. Image compression techniques // International Journal of Computers \& Technology. 2, 2(2012), pp. 49-52.

[16] Cruz, D.; Ebrahimi, T.; Askelof, J.; Larsson, M.; Christopoulos, C. Coding of Still Picture $/ / 45^{\text {th }}$ Proc. of SPIE Applications of Digital Image Processing / 2000.

[17] Chrysafis, C.; Ortega, A. Line-based, reduced memory, wavelet image compression // IEEE Trans. Image Process. 9, 3(2000), pp. 378-389. DOI: 10.1109/83.826776

[18] Fakhari, A.; Fathy, M. A two level architecture for high throughput DCT-processor and implementing on FPGA. // IEEE reconfigurable computing and FPGAs (ReConFig) international conference / Quintana Roo, pp. 115-120.

[19] Mammeri, A.; Hadjou, B.; Khoumsi, A. A survey of image compression algorithms for visual sensor networks. // ISRN Sensor Networks, (2012), pp. 1-19.

[20] Shapiro, J. M. Embedded Image Coding Using Zerotrees of Wavelet Coefficients. // IEEE Trans. on Signal Processing. 41, 12(1993), pp. 3445-3462. DOI: 10.1109/78.258085

[21] Mallaiah, A.; Shabbir, S. K.; Subhashini, T. AnSpiht Algorithm with Huffman Encoder for Image Compression and Quality Improvement Using Retinex Algorithm. // International Journal of Scientific \& Technology Research, 1, 5(2012), pp. 45-49. 
[22] Said, A.; Pearlman W. A. A new, fast, and efficient image codec based on set partitioning in hierarchical trees. // IEEE Transaction on Circuit System Video Technology. 6, 3(1996), pp. 243-250. DOI: 10.1109/76.499834

[23] Sun, E.; Shena, X.; Chena, H.; A Low Energy Image Compression and Transmission in Wireless Multimedia Sensor Networks. // Procedia Engineering. 15, (2011), pp. 3604-3610. DOI: 10.1016/j.proeng.2011.08.675

[24] Huang, W. B.; Su, W. Y.; Kuo, Y. H. VLSI implementation of a modified efficient SPIHT encoder // IEEE Transaction Fundamentals. E89-A, 12(2006), pp. 3613-3622.

[25] Taubman, D. High performance scalable image compression with EBCOT. // IEEE Transaction Image Process. 9, 7(2000), pp. 1151-1170. DOI: 10.1109/83.847830

[26] Ghorbel, O.; Ayedi, W.; Jmal, M. W.; Abid, M. Image compression in WSN: performance analysis. // Communication technology (ICCT) IEEE $14^{\text {th }}$ international conference / Chengdu, 2012, pp. 1363-1368.

[27] Zheng, W.; Liu, Y. Research in a fast DCT algorithm based on JPEG. // IEEE consumer electronics, communications and networks (CECNet) international conference / XianNing, 2011, pp. 551-553.

[28] Wallace, G. K. The JPEG still picture compression standard. // Communications of the ACM. 34, (1991), pp. 18-34. DOI: $10.1145 / 103085.103089$

[29] Nasri, M.; Helali, A.; Sghaier, H.; Maaref, H. Energy efficient wavelet image compression in wireless sensor network // IEEE communication in wireless environments and ubiquitous systems: new challenges (ICWUS) international conference / Sousse, 2010, pp. 1-7.

[30] Nasri, M.; Helali, A.; Sghaier, H.; Maaref, H. Energy conservation for image transmission over wireless sensor networks. // IEEE microelectronics (ICM) international conference / Hammamet, 2011, pp. 1-6.

[31] Jackson, D. J.; Hannah, S. J. Comparative analysis of image compression techniques // IEEE system theory Proceedings SSST Twenty-fifth South-Eastern Symposium // 1993, pp. 513-517.

[32] Mammeri, A.; Khoumsi, A.; Ziou, D.; Hadjou, B. Energyaware JPEG for visual sensor networks. // Proceedings of The 2008 Maghrebian Conference on Software Engineering and Artificial Intelligence, / 2008, pp. 1-7.

[33] Ma, T.; Shrestha, P.; Hempel, M.; Dongming, P.; Sharif, H. Low complexity coder/decoder with an approaching entropy quad tree search code for embedded computing platforms. // Image Processing (ICIP) $18^{\text {th }}$ IEEE International Conference / Brussels, pp. 297-300.

[34] Sun, Y.; Zhang, H.; Hu, G. Real-time implementation of a new low memory SPIHT image coding algorithm using DSP chip. // IEEE Trans Image Process. 11, 9(2002), pp. 1112-1116. DOI: 10.1109/TIP.2002.802533

[35] Kose, K.; Cetin A. E.; Gudukbay, U.; Onural, L. 3D Model Compression using Connectivity-guided Adaptive Lifting Transform. // Signal Processing and Communications Applications SIU 2007. IEEE $15^{\text {th }} /$ Eskisehir, 2007, pp. 14.

[36] Chai, C. W.; Ang, L. M.; Seng, K. P. Multiview image compression for wireless multimedia sensor network using image Stitch in grand SPIHT coding with EZW tree structure // IEEE intelligent human-machine systems and cybernetics IHMSC '09 international conference Hangzhou, Zhejiang, 2009, pp. 298-301.

[37] Xiao, F.; Zhang, P.; Sun, L. J.; Wang, J.; Wang, R. C. Research on image compression and transmission mechanism for wireless multimedia sensor networks. // IEEE electrical and control engineering (ICECE) international conference / Yichang, 2011, pp. 788-791.

[38] Cao, Z. Y.; Ji, Z. Z.; Hu, M. Z. An image sensor node for wireless sensor networks. // International Conference on
Information Technology: Coding and Computing (ITCC 2005 ) international conference, 2005 , pp. 740-745.

\section{Authors' addresses}

Arafat Senturk, PhD. Student

Duzce University, Faculty of Engineering,

Department of Computer Engineering,

Konuralp Campus, Post code 81000, Duzce / Turkey

E-mail: arafatsenturk@duzce.edu.tr

Resul Kara, Assoc. Prof. Dr.

Duzce University, Faculty of Engineering,

Department of Computer Engineering,

Konuralp Campus, Post code 81000, Duzce / Turkey

E-mail: resulkara@duzce.edu.tr 